\title{
Simvastatin-Induced Meralgia Paresthetica
}

\author{
Menahem Sasson, MD, and Shvartzman Pesach, MD
}

3-hydroxy-3-methylglutaryl coenzyme A reductase inhibitors (statins) cause mainly muscular adverse effects. During the last 20 years there has been solid evidence of peripheral neuropathy caused by statins, with a risk of one in 10,000 patients treated for 1 year. Meralgia paresthetica is an entrapment neuropathy occasionally encountered by primary care physicians. To date there has been no report of entrapment neuropathy that could have been caused or aggravated by statins. This case report presents meralgia paresthetica aggravated by simvastatin use that disappeared after simvastatin cessation. ( $\mathrm{J}$ Am Board Fam Med 2011;24:469-473.)

Keywords: Case Report, Entrapment Neuropathy, Meralgia Paresthetica, Peripheral Neuropathy, Simvastatin

Meralgia paresthetica (MP) is an entrapment neuropathy of the lateral femoral cutaneous nerve. It is characterized by a localized paresthesia and numbness on the anterolateral aspect of the thigh. ${ }^{1}$ Its reported incidence is 0.43 per 10,000 person-primary care years per a computerized registration network of a Dutch population study between 1990 to $1998 .^{2}$ Before this, an incidence of 3 cases in 10,000 general clinic patients was reported. ${ }^{3}$

3-hydroxy-3-methylglutaryl coenzyme A reductase inhibitors (statins) have become a dominant lipid-lowering pharmacotherapy ${ }^{4}$ and are widely used. ${ }^{5}$ The best recognized and most commonly reported adverse effects are muscular. ${ }^{5}$ However, during the last 15 years an increasing number of reports and several retrospective cohort studies have been published regarding peripheral neuropathy or polyneuropathy caused by different statins. The incidence is low, although a causal effect was proved, especially after chronic use. ${ }^{6-9}$ Here we

This article was externally peer reviewed.

Submitted 3 October 2010; revised 8 December 2010; accepted 13 December 2010.

From the Department of Family Medicine, Pain and Palliative Care Unit, Siaal Family Medicine and Primary Care Research Center, Ben-Gurion University of the Negev, Beer-Sheva; and Clalit Health Services-South District, Beer-Sheva, Israel.

Funding: none.

Conflict of interest: none declared.

Corresponding author: Dr. Menahm Sasson University of the Negev, Department of Family Medicine Division of Community Health Ben-Gurion, P.O BOX 653 Beer-Sheva 84105 Israel, E-mail: sassonm@bezeqint.net. present an entrapment neuropathy syndrome that seems to be induced or aggravated by simvastatin.

\section{Case}

A 58-year-old white man who was born in Kurdish Iran and currently working as a bus driver presented with a burning pain along the lateral aspect of his right thigh for the past 2 months that worsened when he stood erect. His medical history included asthma, obesity (body mass index, 32), mild hypertension, and hypercholesterolemia. $\mathrm{He}$ denied using alcohol or tobacco and was treated with only a salbutamol inhaler as needed. He had no previous operations. During his physical examination an elliptic hypoesthetic area was identified on the lateral side of his right thigh. A blood count, chemistry with glucose, kidney and liver function tests, and thyroid-stimulating hormone levels were all normal. A month later he returned with an exacerbation of the burning pain to the lateral aspects of both of his thighs (more pain on the right than left), with a pain intensity level of 10 of 10 on a visual analog scale. Again an elliptic hypoesthetic area was diagnosed, though this time on the lateral aspects of both of his thighs. Straight leg raise, muscle strength of lower limb muscles, and patellar and Achilles tendon reflexes were normal. Nonsteroidal anti-inflammatory drugs were offered but were not taken because the patient preferred to consult first with an orthopedist. Electromyography and nerve conduction velocity of the muscles and nerves of the lower limbs gave normal exami- 
nation results for all of the muscles and nerves tested in the lower limbs, except for a low amplitude of the left lateral femoral cutaneous nerve (Figure 1). The patient continued to suffer from the burning pain, especially when standing with an erect posture and after long periods of walking. He was prescribed amitriptyline $20 \mathrm{mg}$ to be taken at night before sleep. The patient reported use of amitriptyline and improvement of the pain during his next visit 2 months later. However, he discontinued the amitriptyline because he could not suffer the sedation side effect.

Figure 1. Electromyography and nerve conduction velocity in the patient's lower limbs. This chart shows normal values of motor and sensory nerve conduction in the lower limbs and electromyelography of the main muscles in the lower limbs. The only abnormal result is a relatively low amplitude $(1.7 \mathrm{uV})$ on the left lateral femoral cutaneous nerve of the thigh. Lat, latency; Amp, amplitude; CV, conduction velocity; F-M, f wave; AHB, abductor hallucis brevis; EDB, extensor digitorum brevis; Stim, stimulation; Gastroc, gastrocnemius; Add, adductor; Fib, fibrillation; PSW, positive sharp waves; Dur, duration; Stabil, stability; Poly, polyphases; Act, action; IP, pattern interference.

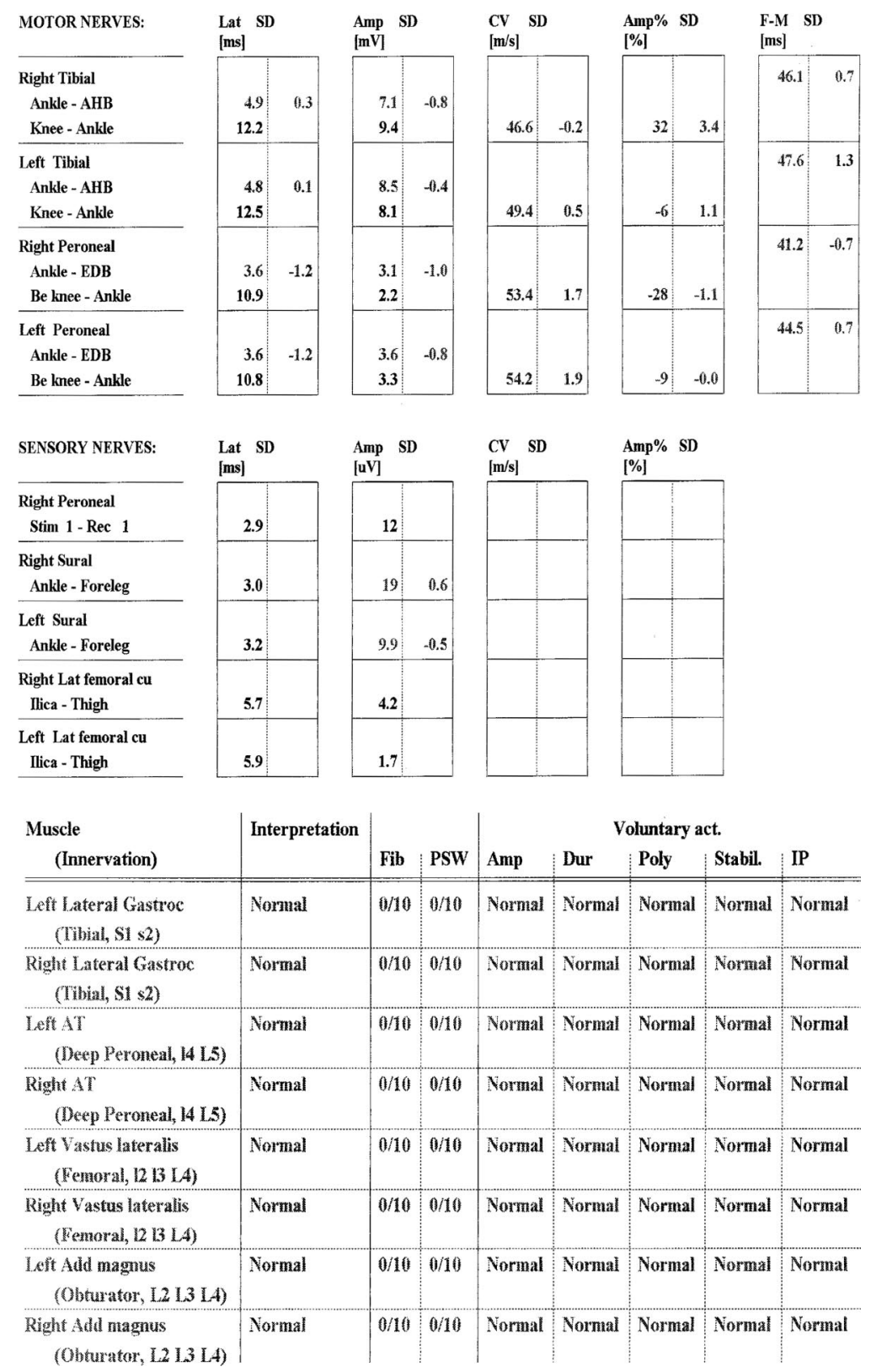


A year after the first encounter the patient returned and reported that he has stopped the use of simvastatin $20 \mathrm{mg}$, which had been prescribed for his hypercholesterolemia about 3 years before. He was afraid of the side effects after reading a newspaper article. The burning pain subsided a week after simvastatin cessation and reached a pain level of 0 of 10 after a month. He was advised to resume simvastatin use at the same dose of $20 \mathrm{mg}$ a day. After a few days of simvastatin renewal the same burning pain began and increased, reaching a pain level of 10 of 10 after 2 weeks. He was advised again to stop simvastatin and the pain decreased again gradually, disappearing completely after a few weeks. Half a year after cessation of the statin use the pain had not returned.

\section{Discussion}

The case presented is typical of MP as described in the literature. Patients usually describe a burning, stinging, or tingling sensation in the thigh and can usually localize the sensation to the skin itself. ${ }^{1}$ The patients do not have back pain, weakness, or reflex changes. ${ }^{10}$ The symptoms are usually unilateral, though $20 \%$ of patients complain about bilateral symptoms that tend to be aggravated by erect posture (as reported by this patient) and prolonged standing. ${ }^{1}$ The lateral femoral cutaneous nerve supplies sensation to a large oval area of skin over the lateral and anterior thigh. ${ }^{10}$ The differential diagnosis includes femoral neuropathy, lumbar plexopathy, and high lumbar radiculopathy. ${ }^{10} \mathrm{Un}$ fortunately, the lateral femoral cutaneous sensory potential is not recorded easily in the electromyography laboratory, even for healthy asymptomatic individuals and especially obese or older individuals. ${ }^{10}$ There are reports that sometimes sensory nerve conduction velocity and somatosensory evoked potentials recorded on the scalp after nerve stimulation can help in the diagnosis, ${ }^{1}$ though not in all cases. These tests are not recommended for routine use. ${ }^{1}$ In our case the sensory nerve conduction velocity of the nerve was studied and a low amplitude of the nerve was observed on the left side, although the patient suffered more from pain on the right side. The other part of the test, which checked the lower limb muscles and nerves, was normal. This supports the exclusion of a femoral neuropathy and lumbar plexopathy or radiculopathy.
Lumbar radiculopathy usually is accompanied by back pain, and the sensory loss is generally ill defined in contrast to the fairly well-demarcated sensory loss from MP. In femoral radiculopathy and lumbar plexopathy, the sensory loss usually involves the entire thigh and extends into the medial thigh and leg. Lumbar radiculopathy, plexopathy, and femoral neuropathy may be accompanied by weakness and a depressed knee jerk, which are not seen with MP. ${ }^{10}$

Most cases of MP are idiopathic, occurring most commonly in patients who are obese; who wear tight underwear, pantyhose, or pants; or who have diabetes mellitus. Certain occupations, such as jobs that require workers to wear heavy tool belts, also can predispose individuals to compression of the lateral femoral cutaneous nerve. MP also can be precipitated by pregnancy. Most cases are caused by entrapment at the inguinal ligament; however, the entrapment can be along the whole course of the nerve originating from the roots of L2-3, crossing the pelvis, and emerging under the inguinal ligament near the anterior superior iliac spine. Thus, tumors or other mass lesions in the psoas, pelvis, or retroperitoneal regions such as abdominal aortic aneurysm, uterine fibroids, or suprarenal tumors have been diagnosed due to MP. ${ }^{10,11}$ Radiologic degenerative pubic symphysis and limb length discrepancy also have been implicated as causes of MP.

Metabolic factors that have been implicated in MP include lead poisoning, alcoholism, diabetes mellitus, and hypothyroidism. ${ }^{1,10,11}$ Iatrogenic causes of MP include occurrence perioperatively or after orthopedic procedures including pelvic osteotomy, spine surgery, laparoscopic cholecystectomy, myomectomy, inguinal hernia repair, coronary artery grafting, aortic valve surgery, gastric reduction surgery for morbid obesity, renal transplant, hip replacement, or iliac crest bone graft harvesting. Rare lesions are caused by direct trauma to the pelvis. ${ }^{1,10}$

Among some patients examined by electromyography, epidemiologic evidence supports previous case reports of statins causing an axonal sensorimotor degeneration neuropathy or a purely small-fiber neuropathy but not demyelination. ${ }^{12,13}$ The peripheral neuropathy or polyneuropathy is characterized by upper and/or lower, proximal, or distal limb weakness, numbness, and pins and needles sensation. ${ }^{12}$ Symptoms 
have been described as beginning from within days to as long as several years after the prescription of statins. ${ }^{14}$ The majority of cases were at least partially reversible with drug cessation. ${ }^{15}$ The risk is increased by longer duration of statin use and the cumulative dose. ${ }^{4,7,9}$ Partial or complete recovery after statin cessation has been described. ${ }^{12,14,15}$ A case of mononeuropathy multiplex and a case of a disorder resembling GuillainBarré also have been described both after simvastatin use. ${ }^{16,17}$

A partial explanation for nerve damage by statins is their inhibition not only of cholesterol synthesis but also of dolichol and ubiquinone; the latter is a key enzyme in the respiratory mitochondrial chain. An intracellular deficiency of ubiquinone has the potential to affect neurons' energy utilization. ${ }^{12}$

In a systematic review from 2006, a total of 16 published case reports and 4 cohort studies of peripheral neuropathy among patients taking statins were identified. There was heterogeneity between the 4 cohort studies regarding the odds ratio of peripheral neuropathy from statins; one study had more extreme result than the others. The combined estimate of odds ratios in these studies (random effect analysis) was 1.8 (95\% CI, 1.1-3.0). ${ }^{4,6-9}$ These results indicate an incidence of peripheral neuropathy attributable to statins of 12 per 100,000 person-years, or a prevalence of 6 per 10,000 persons, ${ }^{8}$ or one in 10,000 patients treated for 1 year. ${ }^{18}$ The risk is low ${ }^{14,19,20}$; however, early diagnosis of this reversible neuropathy will reduce patients' suffering and futile diagnostic tests. Conversely, lately, statin use has been negatively associated with new peripheral sensory neuropathy in a longitudinal study of diabetic patients. ${ }^{21}$

\section{Conclusion}

The use of different 3-hydroxy-3-methylglutaryl coenzyme A reductase inhibitors can increase the risk of idiopathic peripheral polyneuropathy with axonal damage on electromyography. The risk is low, with a combined estimate odds ratio of 1.8, which increases with time and dose of statin use. Statin cessation will reverse the symptoms partially or completely. There is one case report of simvastatin-induced mononeuropathy multiplex and one case report of Guillain-Barré-like neuropathy. This is the first case report of a statin that seemed to cause or aggravate MP, an entrapment neuropathy occasionally encountered in the primary care setting.

\section{References}

1. Harney D, Patijn J. Meralgia paresthetica: diagnosis and management strategies. Pain Med 2007;8:669-77.

2. Van Slobbe AM, Bohnen AM, Bernsen RM, Koes BW, Bierma-Zeinstra SM. Incidence rates and determinants in meralgia paresthetica in general practice. J Neurol 2004;251:294-7.

3. Ecker AD. Diagnosis of meralgia paresthetica. JAMA 1985;253:976.

4. Anderson J, Muhlestein JB, Bair T, et al. Do statins increase the risk of idiopathic polyneuropathy? Am J Cardiol 2005;95:1097-9.

5. Golomb BA, Evans MA. Statins adverse effects: a review of the literature and evidence for a mitochondrial mechanism. Am J Cardiovasc Drugs 2008;8(6): 373-418.

6. Gaist D, Garcia Rodriguez LA, Huerta C, Hallas J, Sindrup SH. Are users of lipid-lowering drugs at increased risk of peripheral neuropathy? Eur J Clin Pharmacol 2001;56:931-3.

7. Corrao G, Zambon A, Bertu L, Botteri E, Leoni O, Contiero P. Lipid lowering drugs prescription and the risk of peripheral neuropathy: an explanatory case-control study using automated database. J Epidemiol Community Health 2004;58:1047-51.

8. Law M, Rudnicka AR. Statin safety:a systematic review. Am J Cardiol 2006;97:52C-60C.

9. Gaist D, Jeppesen U, Andersen M, Rodriguez G, Hallas S, Sindrup SH. Statins and risk of polyneuropathy. Neurology 2003;58:1333-7.

10. Shapiro BE, Preston DC. Entrapment and compressive neuropathies. Med Clin North Am 2009;93: 285-315.

11. Ivanez V. Meralgia paresthetica is not always an entrapment neuropathy. J Fam Pract 2004;53:420.

12. Phan T, McLeod JG, Pollard JD, Peiris O, Rohan A, Halpern JP. Peripheral neuropathy associated with simvastatin. J Neurol Neurosurg Psychiatry 1995;58: 625-8.

13. Peltier A, Russell JW. Advances in understanding drug-induced neuropathies. Drug Saf 2006;29:2330.

14. Weimer LH, Sachdev N. Update on medicationinduced peripheral neuropathy. Curr Neurol Neurosci Rep 2009;9:69-75.

15. Backes JM, Howard PA. Association of HMGCoA reductase inhibitors with neuropathy. Ann Pharmacother 2003;37:274-8.

16. Scola RH, Trentin AP, Germiniani FM, Piovesan EJ, Werneck LC. Simvastatin-induced mononeuropathy multiplex: case report. Arq Neuropsiquiatr 2004;62:540-2.

17. Rajabally YA, Varakantam V, Abbott RJ. Disorder resembling Guillian-Barre syndrome on initia- 
tion of statin therapy. Muscle Nerve 2004;30: 663-6.

18. Peripheral neuropathy and statins. Prescrire Int 2007;16:247-8.

19. Brown W. Safety of statins. Curr Opin Lipidol 2008; 19:558-62.
20. Formaglio M, Vial C. Statin induced neurpathy: myth or reality? Rev Neurol 2006;162:1286-9.

21. Davis TME, Yeap BB, Davis WA, Bruce DG. Lipidlowering therapy and peripheral sensory neuropathy in type 2 diabetes: the Fremantle diabetes study. Diabetologia 2008;51:562-6. 